\title{
Towards a Needs Analysis of why Students in a Ghanaian University Fail the Academic Writing Course
}

\author{
Nartey, Mark \\ Department of Language and Literature, Norwegian University of \\ Science and Technology Trondheim, Norway \\ Email:narteynartey60@gmail.com \\ Dorgbetor, Nathaniel \\ Department of Language and Literature,Norwegian University of \\ Science and TechnologyTrondheim, Norway \\ Email: kwekunat@gmail.com
}

\section{Doi:10.5901/jesr.2014.v4n6p167}

\begin{abstract}
In the last two and half decades, academic literacy has received considerable attention in tertiary education in several Englishmedium universities. Consequently, English for Academic Purposes (EAP) and analogous writing programs have constantly been revised in the United States of America, the United Kingdom and Australia, especially with the objective of meeting the needs of students. Contrarily, EAP programs in most countries in sub-saharan Africa, including Ghana have received very little innovation and change. In this paper, we moot the idea that the principal reason for which a vast majority of students fail an EAP program (Academic Writing) in an English-medium university in Ghana stems from the lack of correlation between the course curriculum/classroom pedagogy and the course examination. To this end, we propose key changes to the curriculum, and conclude with a discussion of some implications worth considering for both curriculum designers and implementers of EAP in university education.
\end{abstract}

Keywords: Academic writing. Ghana, students, university

\section{Introduction}

In the last three decades, studies on academic literacy have continually shown that the nexus between academic literacy and tertiary education is undeniable (Lea and Stierer 2000; Afful 2007; Nartey and Coker 2011). Not surprisingly, academic literacy and tertiary education have long engaged the attention of educationists, applied linguists and other scholars interested in the use of language by tertiary education students. Unlike in non-native settings, these issues have gained considerable momentum and have been discussed by scholars in American, British, Australian and Canadian universities, notwithstanding the fact non-native countries continue to use English not only in academic spheres but also in business, political and sometimes even in social domains as a result of globalization and the concomitant use of English as an international language (Block and Cameron 2003).

Generally, the considerable attention that has been paid to academic literacy worldwide in the last three decades as Afful (2007: 141) rightly notes "derives from the challenges posed by globalization, internationalization and the increasing prominence given to English language education globally. The term 'academic literacy' is a composite of the generic, transferrable skills that are required of and developed by academic study and research, and which enables one to function effectively in various disciplinary communities in a university. Essentially, these skills include knowledge of how academic discourse is structured and produced, creative and critical thinking, independent learning, among others.

In many English-medium universities, a key course that is taught to facilitate and/or enhance the acquisition of academic literacy skills is English for Academic Purposes (EAP). In second language contexts, such as the Ghanaian situation, the objectives of EAP are contained in Communicative Skills and in recent times Academic Writing, a threehour mandatory course read by all first year students in all public universities. This course is premised on the empirical assumption that high school students entering university are seen to have a culture, practices and values different from those of universities or tertiary institutions (Alfers and Dison 2000; cited in Afful 2007: 142). That is, university students are expected to demonstrate express knowledge of writing in specific genres for specific communicative endeavors, a 
task they may have hardly been exposed to at the pre-tertiary level.

Over the years, studies in academic literacy, in general, and EAP, in particular, (MacDonald 1994; Jordan 2000; Zhao 2004; Turner 2004) have garnered scholarly attention in countries like the US, UK and Australia, culminating into substantial changes in pedagogy, advances in technology, and new emphasis on critical reading, writing, and thinking across the curriculum. Unlike these countries, EAP programs in tertiary institutions in Ghana have not witnessed much systematic evaluation. In this paper, we explore the discrepancy between curriculum and exams of an EAP course (Academic Writing) in an English-medium university in Ghana, arguing that the principal reason why a vast majority of students fail the course stems from the lack of correlation between the curriculum and the exams.

To accomplish this task, we first describe the general landscape of the teaching of EAP and similar writing programs in English-medium universities. Second, we foreground the teaching of Academic Writing in our educational setting, placing it within the geo-political context of English language education in Ghana. Third, we provide a twopronged rationale for changes in the Academic Writing curriculum and follow it up with the proposed changes. The implications for theoreticians and implementers of EAP in university education are finally discussed.

\section{Teaching of English for Academic Purposes (EAP)}

Currently, EAP occupies a central role in English-medium universities and universities across the globe. The growth of EAP is derived from the awareness of ESP practitioners that tertiary level students possess different learning needs which cannot be met by teaching them the same type of English language. Essentially, the existence of EAP or analogous writing programs is predicated on one fundamental assumption often found in the literature: writing at preuniversity level is markedly different from the writing required at the tertiary level (Alfers and Dison 2000; Afful 2007).

For instance, Sabariah and Rafik-Galea (2008) view the development of EAP as a result of dissatisfaction with the generalizability of ESP courses. Evans and Green (2007), in a study conducted in Hong Kong to find out the tertiary students English language needs, found that most of the undergraduate students "not only required language support at university, but also that this support should be oriented towards academic rather than general English" (p. 5). Hyland (1997) also notes that students generally see the value of EAP classes as they recognize that proficiency in English is an important determinant of academic success in an English-medium environment.

Hitherto referred to as English for Educational Purposes (EEP) (Shing and Sim 2011), EAP is often given different designations in different countries. In the USA, it is labeled Writing in the Disciplines whereas in the UK and Canada, it is often referred to as English for Academic Purposes (EAP). Mainly, the promotion of EAP and other allied programs in these native settings stemmed from the increasing internationalization of tertiary education (Jordan 2002). In this regard, it may be argued that instituting EAP programs in Africa, Asia, Latin America and Europe is inevitable, given the international character of universities and the more cogent reason of the increasing role of English as an "academic lingua franca" (Duszak 1997: 21).

The importance of EAP cannot be overemphasized as it "assumes a preparatory, facilitative and catalytic role for fresh students, ensuring the smooth transition from pre-university stage to the university level" (Afful 2007: 143). Unfortunately, this pivotal role of EAP is sometimes branded in the literature with reductionist and derogatory terms as 'remedial', 'adjunct' or 'periphery'. This assumption, notwithstanding, there is no gainsaying the point that writing programs serve different needs in order to equip them to undertake various academic tasks and to engage in activities in the university, even if minimally.

An important point worth mentioning is that EAP is sometimes considered along two strands: English for Specific Academic Purposes (ESAP) and English for General Academic Purposes (EGAP). Describing EGAP, Jordan (1997: 5) avers that "a large proportion of the common core element is more usually known as 'study skills', plus other elements of a general academic English register, incorporating a formal academic style with proficiencyin language use. He adds that these study skills include effective lecture listening, comprehension and note-taking, writing in the appropriate academic register, reading effectively for study purposes, participation in discussion and library research. In ESAP, however, vocabulary and skills specific to a subject of study are emphasized. This distinction between EGAP and ESAP presented above is reiterated by Dudley-Evans and St. John (1998) when they opined that the dichotomy between ESAP and EGAP is that ESAP courses focus on the actual tasks that students have to carry out while EGAP courses select more general contexts.

A close look at EAP programs in non-native settings reveals at least two interesting findings: the labels by which they are identified and their content. In India, for instance, many universities prefer the term Communication Skills, while in Hong Kong, the situation is quite fuzzy: while several universities employ $E A P$, a few use English for Communication 
Purposes. In Singapore, most tertiary institutions prefer to use the term EAP or other ESP tradition terms such as English for Business or English for Engineering, inter alia, in a bid to situate the writing program in specific disciplinary milieus. Generally, universities in Africa use labels such as Communicative Skills, Communication Skills, Use of English and in recent times Academic Writing. Expectedly, these differences in labels imply differences in curricular, pedagogy and even philosophical orientations. For example, in terms of curricular, most EAP programs in African and Indian universities emphasize the written aspect. Conversely, universities in Singapore usually follow British models by incorporating speaking and listening to a large extent.

\section{The Nature of Academic Writing in a Ghanaian University}

Ghana's contact with Europeans - the Portuguese, the Dutch and the British - has spanned over three centuries. These long years of contact notwithstanding, it is the English language that has exerted much influence over Ghana, one of the countries in the world where English is the only official language and arguably a somewhat 'national' language. Although the English language co-exists with over forty indigenous languages (KroppDakubu 1988), English attracts the attention of everyone, given its wide range of application in the domestic affairs of Ghana (used for example in governance, education, media, law, commerce, etc.) as well as in international communication and science. The centrality of English in the public domains of life in the country is summarized by Sarfo (2011: 460) when he avers that "English language has come to stay as a communicative tool for social, political and economic development".

As far as education is concerned, English serves as the medium of instruction in Ghanaian universities, including the University of Ghana (UG), the institutional setting and educational context for this study. A public university established in 1948, UG conducts its teaching, learning and research through two principal colleges, twenty one research centers and six faculties: Arts, Law, Sciences, Social Studies, Business school and Engineering Science, enabling it to provide a number of academic programs to over 30, 000 local and international students. The choice of UG is informed by our familiarity with its academic and non-academic members, coupled with the fact that we are abreast of the modus operandi of the institution. Like other Ghanaian public universities, English (as well as Mathematics and Science) remains a compulsory requirement for entry into UG. Besides, prospective university students in Ghana are expected to have been exposed to twelve years of English from the primary school level to the senior high school level. Still, on admission into Ghanaian universities, students are required to take Academic Writing, or in other jurisdictions, Communicative/Communication Skills.

Generally, the Academic Writing course in UG is two-pronged, underscoring remediation and writing/communication skills. A vignette of the curriculum is presented below.

- The nature of academic writing

- Basic issues in grammar

- Sentence structure and punctuation

- Strategies for vocabulary development

- Paragraph structure

- Methods of paragraphing and development

- Referencing skills, documentation skills and avoiding plagiarism

- Deviant usage

The primary objective of the Academic Writing course in UG is to equip students with the writing/communication skills that would enable them to succeed in the academic discourse community. Essentially, thus, the course focuses on enhancing the quality of language use of students. Related to the Academic Writing course are issues such as the teaching staff, allocation of credit hours and writing guides. The course is a three-hour one and is taught over two years: Academic Writing I in the first year and Academic Writing II in the second year. The course is taught by Faculty members on part-time basis; these course instructors are not necessarily members of the Language Centre, the outfit responsible for mounting the course. To facilitate the teaching and learning of the course, handouts are provided by the Course Coordinator's Office and other materials deemed needful by various instructors are also used.

Suffice it to say that the course is compulsory for all students in UG and they are required to pass it before they can be awarded their certificates on completion of their university education. The underlying premise of Academic Writing as a foundational course is that language skills can be decontextualized from content and that academic language, though rooted in specific disciplines, has common features across disciplines. 


\section{The Case for a Change in the Academic Writing Curriculum at UG}

In this section, we argue for a change in the curriculum of Academic Writing at UG based on two principal issues: the poor performance of students and the lack of correlation between classroom pedagogy and the course examination.

The first cardinal reason for proposing a change in the University of Ghana's Academic Writing Curriculum is the poor performance of students stemming from the lack of correlation between the course content and/or curriculum and the course examination. Available evidence lends credence to the view that usually a greater number of students in UG who take the course fail: for example, in the 2011/2012 academic year where more than half of the students who took the course failed, i.e. they scored below the 50 per cent pass mark. And it is our view that this alarming rate of failure cannot necessarily be attributed to the non-performance of students, but to the absence of a good relationship between the actual content of the course as delivered in the classroom and the nature of the examination questions.

Generally, the course's curriculum, as previously noted, states explicitly that the main objective of the course is to equip students with the communication skills that would enable them to succeed in the academic discourse community.To achieve this objective, the course description suggests that students are taken through strategies for paragraph development, study reading, gathering and using information from library and other sources, integrating information into essays (i.e. summarizing as part of exposition and writing citations) all within the larger context of enhancing the quality of language use of students. While this objective and the strategies for achieving it may have been well spelt out in the curriculum, the examination for the course, given its nature, does not reflect the course content to a large extent.

A quick scan of the examination questions shows that the bulk of the paper is centered on testing the summary skills of the students and not necessarily their ability to write academically. The role of summary writing to the examination is so critical that the remainder of the paper, the composition,is inextricably linked with it since students are required to write a composition by culling evidence from the same passage usedfor the summary. Following from this, it will not be far-fetched to intimate that the composition part of the examination is another summary in disguise since the students are expected to incorporate a vast majority of ideas from the summary passage into their own essays. Sometimes, some few questions on basic grammar and general questions like "what is plagiarism or what is an academic discourse community?" are also asked in addition to the summary and composition.

Closely studying the examination questions vis-à-vis the curriculum, it is discernible that these two components of the course do not seem to be in consonance with each other: while the curriculum focuses on skills development, the examination does not allow for such skills to be fully tested and/or assessed. It is obvious that the key determinant of whether or not a student passes the examinationis the summary part of the paper, especially when more than half of the total marks is allocated to it. While conceding that a student's ability to effectively summarize information from different sources is quintessential in academia, we argue that the curriculum and the actual course content and/or course description does not place such premium on summary writing.

Given this apparent discrepancy between the curriculum and the examination, it is our view that since the curriculum does not give such special priority to summary writing, then the examination should do same. Better yet, the curriculum could be morphed to reflect this overarching importance of summary writing in the examination. Besides, it is our observation also that the passages from which the students are required to write their summaries, more often than not, are detached from the immediate or even remote environments of the students. Subsequently, the students are unable to connect or identify with the passages, thereby preventing them from effectively applying the summary skills acquired.

The second reason for advocating a change in the curriculum is the lack of correspondence between classroom pedagogy and the examination, which invariably contributes to the poor performance of students in the course. In this regard, our observation reveals that the teaching and learning methodology adopted by some course instructors when juxtaposed with the nature of the examination leaves a lot to be desired. That is, some of them overly foreground some aspects of the curriculum, albeit those aspects may not necessarily be needful for the examination. As discussed above, the examination for the Academic Writing course in UG mainly highlights summary writing and composition to a lesser extent. In view of this, one would expect that the pedagogical procedures employed in the classroom will also place special attention on these areas. Indeed, it would be expected that hands-on exercises and assignments on summary writing and composition will be a common feature in the Academic Writing classroom in UG. Unfortunately, this is not the case.

Instead, different lecturers foreground different areas of the course, perhaps, depending on their personal interests and strengths. Most course instructors, for instance, focus much attention on how to make academic presentations, 
which in all fairness to them is a good skill to acquire. Meanwhile, the examination does not comprise making academic presentations.Granted, knowledge acquisition is not all about getting ready for and passing an examination. However, if such knowledge is overly emphasized at the expense of other equally important things which are relevant for an examination, that, to our minds, does not augur well for a student's overall academic development.

Ultimately, owing to this lack of correspondence between classroom pedagogy and the examination, students are most likely to be ill-prepared for the examination. In the light of the above, we submit that there is the need for the curriculum to be tinkered with in order to guide which methodological procedures are deployed by course instructors in the classroom, and more importantly, for these procedures to be in tandem with the nature of the course examination.

\section{The Proposed Changes}

Our proposed changes for the Academic Writing course are two - fold: first, a change in the course content or a reform in the examination format; second, a change in methodological/pedagogical approach, especially as regards the theme based approach.

\subsection{A Change in the Course Content or a Reform in the Examination Format}

Fundamentally, we propose a change in the actual content of the course and/or a reformation in the format of the examination so that ultimately these two vital components of the course - which are inextricably linked - compensate each other or are a true reflection of each other. Undoubtedly, these two aspects of the course move hand-in-hand, and it is therefore expected that they complement each other. Such a correlation between the course content and the examination, we reckon, will present a more pragmatic means of assessing the performance of students. As well, we maintain that a fine correlation between these two core aspects of the course has a very high potential of improving upon the performance of students in the course.

Our proposed change in the course content or a reform in the examination is predicated on the role of Academic Writing and/or Communicative Skills as a foundational or remedial course in tertiary education in Ghana (Afful 2007). With regard to the foundational perspective, Academic Writing or Communicative Skills seeks to be an empowering force that enables students to function effectively in the university community in terms of their ability to perform various academic tasks. Conversely, the remedial stance seeks to deal with the language deficiencies of students. Clearly, these two principles on which our proposal for a direct relationship between the course content and the examination format is hinged are critical for the overall language skills development of the students. In view of this, the poor performance of students stemming from a disconnection between the course content and the examination format need not be treated lightly.

Studying the course content of the first year students, we find that the overarching focus is on issues of grammar: sentence structure, punctuation and vocabulary development, among others. Meanwhile, the format of the examination is such that summary writing and composition are fore-grounded. Given this discrepancy, we propose that the issues of grammar which may not necessarily be essential to the examination be given less attention in the curriculum or course content. Besides, it is our view that it is partly redundant to have Basic Issues in Grammar as a major topic in the curriculum and still have topics such as Sentence Structure and Punctuation also as major topics. This is because these latter topics can neatly fit in or be subsumed under the much broader topic of Basic Issues in Grammar.

The danger of considering all these topics as major topics is that there is a high propensity for course instructors to spend much time on them (as separate major topics) at the expense of other relevant topics. Meanwhile, these topics are not extensively represented in the examination. In the light of the above, we propose that Sentence Structure and Punctuation be treated as minor topics under Basic Issues in Grammar. Still, Vocabulary Development as another major topic in the curriculum of the first year students, we propose, should be treated in relation with the composition component of the course. Since the linguistic repertoire of students is only indirectly tested through mainly the composition component of the examination, we argue that it will be a misplaced priority to consider vocabulary development as a separate major topic.

Two changes are vital as far as the topics in the curriculum of the second year students are concerned: Deviant Usage and Making Academic Presentations. These two topics, especially the latter, do not quite reflect the principal objective of the course, which is to equip students with the writing skills that would enable them to succeed in the academic discourse community. While admitting that the topic Deviant Usage (which highlights common grammatical infelicities and linguistic inexactitudes of users of English) is useful for academic writing, in general, this remediation 
strand of language use - it is assumed - had been taken care of in the first year curriculum of the course under the topic Basic Issues in Grammar. A repetition of the topic - albeit couched differently - is unnecessary, especially when the topic is not critical to the overall performance of students in the course. This is because these deviant forms are only minimally assessed in the examination.

In a bid to improve upon the performance of students in the Academic Writing course, we intimate that it is imperative for the examination format to explicitly and/or implicitly reflect the course content. To this end, we propose the following changes to the examination.

For the first year students, the summary component of the examination should be scrapped. This is because their curriculum does not entail summary writing, and yet it forms almost half of the total marks of the paper. As previously indicated, the primary focus of the curriculum of the Academic Writing course for the first year students is issues of grammar. Following this, we propose that the overwhelming emphasis on issues of grammar in the curriculum should similarly be translated into the examination. Therefore, basic issues that border on grammar, including sentence types/structure, punctuation and deviant usage (which are largely captured in the curriculum), should feature prominently as well in the examination.

As far as the second year students of the course are concerned, we propose, first and foremost, that definitional questions should be scrapped since such questions merely demand 'reproductive' answers/knowledge from students, and so do not necessarily test practical knowledge. Examples of such definitional questions include "what is academic writing/plagiarism, etc.?" Second, questions that border on deviant usage should also be scrapped since such questions would have been taken care of in the first year of the course. Third and more crucially, the examination questions should focus on summary writing and composition in order to assess the extent to which students are able to apply or incorporate knowledge acquired from both the first and second years of the course in their writing.

Again, we propose that the composition part of the examination for the second years should not necessarily be linked to the passage used for the summary as is often the case. Such an approach, to our minds, is delimiting and, subsequently, can restrict students in their ability to imagine and brainstorm - this can adversely affect their performance. Rather than strictly associate the composition topic to the passage used for the summary, students should be given a minimum of two different topical areas on which their compositions can be based. When given this 'open' option, students will be afforded the opportunity to freely express their views on a subject matter - this has a high potential of improving upon their performance.

\subsection{Change in Methodological/Pedagogical Approach}

In addition to a change in the course content and/or a reform in the examination format, we propose a change in the methodological/pedagogical approach adopted in the Academic Writing classroom. To this end, we submit that the instruction in the classroom should largely (and not necessarily exclusively) focus attention on examinable content. As well, there should be practical exercises/assignments, especially on summary writing and composition as well as other hands-on activities that will adequately prepare students for the examination. Further, we propose a change with respect to the theme-based approach (with specific focus on topics selected for passages) adopted in the classroom.

The need for the teaching approach in the Academic Writing classroom to focus much attention on examinable content is imperative, especially when our observation reveals that some course instructors spend much time on less examinable content at the expense of more likely examinable content. As previously indicated, some course instructors, for instance, overly foreground some aspects of the curriculum which may not necessarily be needful for the examination: such a practice should be discouraged since it has a direct negative impact on the performance of students.

Generally, the course is taught for 13 teaching weeks. Out of these 13 weeks, it is our observation that Summary Writing is usually taught for a week and How to make an Academic Presentation is taught within 3 to 4 weeks depending on the size of a class. In view of this, the summary component of the course is likely to be taught hastily. Meanwhile, in the examination, the ability of a student to make an academic presentation is neither assessed directly or indirectly despite the huge amount of time that is invested in teaching the topic. Conversely, summary writing (which is sparsely treated during the course of the semester) forms nearly half of the total marks allocated for the paper.

To our minds, this situation does not augur well for students' preparation for the examination. Consequently, they are likely to perform below expectation. Besides the consumption of man hours needed for other aspects of the course that form an integral part of the examination, such imbalance between the course distribution and the examination could adversely affect students' preparation for the examination psychologically. For instance, most students are likely to concentrate a lot more on their personal presentation skills or on some techniques of presentation, etc. in a bid to pass 
the examination. If on the day of the examination, however, they come to the realization that such a topic - the huge amount of time expended on it notwithstanding - does not feature at all in the examination, the resulting dismay could negatively affect their approach to the entire paper. Following from the above, we suggest that rather than academic presentation, 3 or 4 weeks should be spent on summary writing, or at least there should be an even distribution in the time allocation.

The role of practical exercises/tasks to any teaching and learning methodology can neither be underestimated nor overemphasized. Such exercises afford students the opportunity to test the extent of knowledge acquired and, subsequently, aid them in their examination preparation. It should therefore be a thing of concern if such exercises do not feature prominently in a course such as Academic Writing. Undoubtedly, the art of writing is perfected through constant and conscious practice. We maintain that teaching students how to write compositions can be challenging and, perhaps, boring even so when the students are not actively engaged in regular exercises.

Admittedly, the rather large classes of the Academic Writing course in UG may not encourage course instructors to organize enough exercises and assignments. Nonetheless, the point still holds that the absence of such practical tasks could get students ill-prepared for the examination. Given this, we propose that as a sure means of improving upon the performance of students in the course, hand-on exercises - especially on summary writing and composition - should be integrated into the classroom instruction. Essentially, these exercises would help instructors monitor the steady progress of students and make the necessary adjustments to their instructional approach. Take-home assignments should also be given at regular intervals. Hands-on exercises and assignments on summary writing and composition will not only help students prepare adequately for the examination, but also equip them with requite writing-specific skills as stated in the course compendium.

On the theme-based approach, our observation, as already indicated, show that the passages from which the students are required to cull their summaries are more, often than not, detached from the immediate environment of the students. Consequently, the students are unable to connect or identify with the passages, thereby preventing them from effectively applying the summary skills acquired. Owing to this, we suggest that the passages selected for the summary during classroom exercises and take-home assignments, for instance, should not be based on issues removed from both the immediate and remote environments of the students. By so doing, the students will be able comprehend the passages more easily, and be able to effectively apply the summary skills acquired.

A theme-based approach to the selection of passages meant to highlight various aspects of a topic has been proven to be successful in generating and maintaining interest in reading among students in South African universities. For instance, Afful (2007) notes that in post-apartheid South Africa, passages that are chosen in EAP programs are a depicture of issues of national concern: for example, diversity of races, equality, violence, HIVIAIDS, etc. Afful adds that these topical national issues "have the advantage of holding the attention of students and facilitating their understanding of pertinent issues on academic writing" (p.152). Similarly, we advocate for a theme-based approach in the selection of passages for the Academic Writing course in UG: such an approach could take into serious consideration socio-cultural, religious, political and historical circumstances of Ghana in order that passages that deal with festivals, rites of passage, gender, language use, sports as well as gender will be selected.

Importantly, we suggest that the teaching methodology for the course should be explicitly spelt out as part of the curriculum so that a certain level of balance is ensured across the board as far as the numerous course instructors of the course are concerned.

\section{Conclusion}

In this paper, we have examined the possible reasons underpinning the poor performance of students in an EAP program in a Ghanaian university. Using the course curriculum and the course examination, we set out to show how a disconnection between these two vital components of the course can impact negatively on the overall performance of students. Largely, the study points to the view that the poor performance of students in the Academic Writing course in UG stems from the lack of correlation between classroom pedagogy/course curriculum and the course examination. Owing to the 'needs' analysis presented in this study, certain changes to both the course curriculum and course examination were mooted.

Evidently, the study holds two major implications. At the level of curriculum, it is hoped that course designers of Academic Writing in UG reconsider the nature of the course, its content and possibly the mode of assessment. This perhaps stems from the generally-held view that there is a wide gap between the demands of pre-tertiary education visà-vis the task expected of students at the university. In respect of pedagogical approach, it is only normal to expect that 
the methodological procedures adopted in the classroom will adequately compensate and/or complement the curriculum. Perhaps, the curriculum could also encourage teaching adaptability in the classroom.

\section{References}

Afful, J.B.A. (2007). Academic literacy and communicative skills in the Ghanaian university: A proposal. Nebula, 4(3): 141-159.

Alfers, H. \& Dison, A. (2007). Tracking students through the system: An investigation into EAP students' progress at Rhodes University. Proceedings of the SAALA 2000 Conference, 1: 1-10.

Block, D. \& Cameron, D. (eds.) (2002). Globalization and language teaching. London \& New York: Routledge.

Dudley-Evans, T. \& St. John, M.T. (1998). Development in English for specific purposes: A multi-disciplinary approach. Cambridge: Cambridge University Press.

Evans, S. \& Green, C. (2007). Why EAP is necessary: A survey of Hong Kong tertiary students. Journal of English for Academic Purposes, 6: 3-17.

Hyland, K. (1997). Is EAP necessary? A survey of Hong Kong undergraduates. Asian Journal of English Language Teaching, 7: 77-99.

Jordan, R.R. (1997). English for academic purposes: A guide and resource book for teachers. Cambridge: Cambridge University Press.

Jordan, R.R. (2002). The growth of EAP in Britain. Journal of English for Academic Purposes, 1(1): 69-75.

Kropp Dakubu, M.E. (1988). The languages of Ghana. London: Keagen Paul International for the International African Institute.

Lea, M.R. \& Stierer (eds.) (2002). Student writing in higher education: New contexts. Buckingham: Society for Research into Higher Education and Open University Press.

MacDonald, S.P. (1994). Professional academic writing in the humanities and social sciences. Carbondale: Southern Illinois University Press.

Nartey, T. \& Coker, C. (2011). A preliminary report on first year university students' knowledge of basic grammar: the case of the University of Cape Coast. Department of Communication Studies. Unpublished.

Sabariah, M.R. \& Rafik-Galea, S. (2005). Designing test specifications for assessing ESL writing skills in English for academic purposes. In: S.H. Chan \& M.E. Vethamani (eds.) ELT Concerns in Assessment. Pp.149-167, Petaling Jaya: Sasbadi Sdn. Bhd.

Sarfo, E. (2011). English language and sustainable development in Ghana. Language in India, 2011(1): 460-469.

Turner, J. (2004). Language as academic purpose. Journal of English for Academic Purposes, 3: 95-109.

Zhao, F. (2004). Commercialization of research: A case of Australian universities. Higher Education Research \& Development, 23(2): 223-236. 